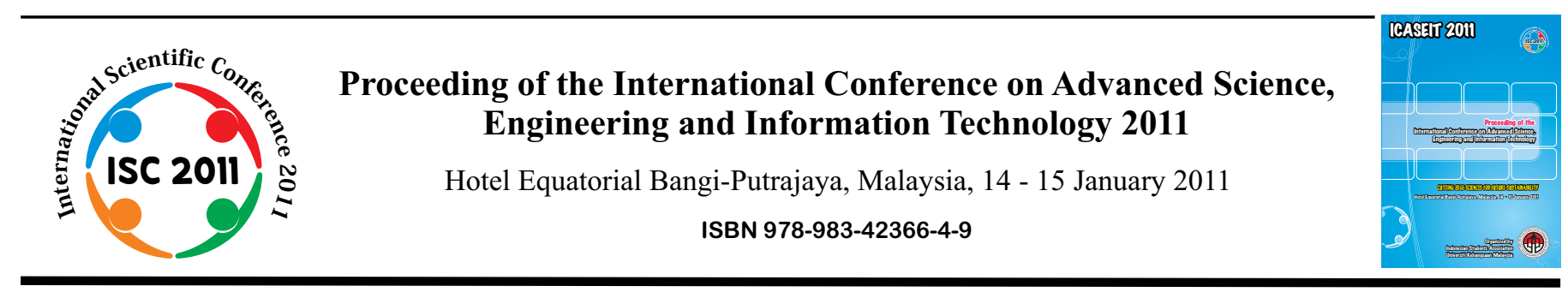

\title{
Applications of Computer Aided Design (CAD) in Medical Image Technology
}

\author{
Azrulhizam Shapi'i ', Riza Sulaiman",Mohammad Khatim Hasan", \\ Abd Yazid Mohd Kassim*, Hamzaini Abd Hamid* \\ \# Industrial Computing Research Group, Faculty of Information Science and Technology \\ Universiti Kebangsaan Malaysia, 43600 Bangi, Malaysia \\ Tel.:+60389216180, E-mail: azrul@ftsm.ukm.my
}

*Department of Orthopaedics and Traumalogy,Medical Centre of Universiti Kebangsaan Malaysia

Bandar Tun Razak, 56000 Cheras, Kuala Lumpur, Malaysia

E-mail: dryazidk@gmail.com

\begin{abstract}
This paper is submitting the idea of Computer Aided Design (CAD) software application in manipulation of digital x-ray images (DICOM). The study also discusses the concept of raster and vector images as DICOM images will be referred to as raster graphic and CAD as vector graphic. Vectorizing allows an image to be more flexible and can be manipulated so that more information can be loaded into it. As such, it is not impossible that vectorizing method can also be performed on medical images using CAD software such as AutoCAD, Solidworks and others. A DICOM image with DCM format is converted to JPEG format using Medweb software. Then, by using Image2CAD software, the $x$-ray image is converted to DXF format. The results showed that patient's x-ray image can be manipulated by using AutoCAD software. Study shows that CAD is not only used in the manufacturing field, but it can also be used in the medical field as well.
\end{abstract}

Keywords - Computer Aided Design, vector, medical image, manipulate, $x$-ray

\section{INTRODUCTION}

The medical field is currently using the Information Technology (IT) application especially in communications and imaging of scanned images such as X-ray and Computerized Tomography Scans (CT Scans). These images require a specific format intended for storage that can be achieved by a few of workstations in a medical organization. One of the most commonly used formats is the Digital Imaging and Communication in Medicine (DICOM). This standard supports the concept of two-dimensional (2D) and three-dimensional (3D) [1].

In other areas such as industries involved in the design of product development, the most popular format used is the drawing (*.dwg). The field is also expanding with the usage of computer applications such as Computer Aided Design (CAD) in designing a product. This allows for easier design process and more effective than paper drawing [2]. Furthermore, the design can be done in three dimensions.

This study is to submit ideas of Computer Aided Design (CAD) software in manipulating medical images (DICOM).
The study also discusses the concept of raster and vector images as DICOM images will be referred to as raster graphic and CAD as vector graphic.

Raster image or better known as raster graphic is often referred to as a bitmap which means data file or general structure represents a rectangular grid of pixels, or dots of colour on a computer, paper or other display device [3]. The colour of each pixel is defined in its own image of the RGB colour space. For example, for a colour pixel, it is defined by three bytes and each byte represents red, green and blue. The less colour images require less information per pixel. For black and white image of each pixel requires only one bit [4]. Raster image is different from vector graphic that represents the image through geometric object usage such as curves and polygons. The raster image coordinates the image displayed on the screen bit by bit and probably with the same format as the one that is to be stored in video display memory [3]. Examples of raster image is the illustrated letter ' $\mathrm{J}$ ', the vision at close range will appear as in figure 1 and figure 2 , where the characters ' $\mathrm{X}$ ' and '. ' will be read by 
the computer as '.' representing ' 0 ' and the characters ' $\mathrm{X}$ ' representing 1 .

$$
\begin{aligned}
& \ldots \ldots x \\
& \ldots \ldots x \\
& \ldots \ldots x \\
& \ldots \ldots x \\
& \ldots . x \\
& \ldots \ldots x \\
& X \ldots x \\
& X \ldots x \\
& . X x x x .
\end{aligned}
$$

Fig. 1 Raster Image

000001
000001
000001
000001
000001
000001
100001
100001
011110

Fig. 2 Image as read by the computer

Vector graphics (also called geometric modeling or objectoriented graphics) is the use of geometrical primitives such as points, lines, curves and polygons where we use a collection of pixels to represent image. Presently, computer display translates the representation of vector image to raster format. Vectorizing is important because it will be used to remove unnecessary details from a photograph. Vectorizing applications is normally used in GIS (Geographical Information Systems) to convert mapping image into a vector format for the purpose image manipulation.

\section{OBJECTIVES}

The objective of this study is to review the two types of data format in different areas namely DICOM and CAD and to convert DICOM images to CAD format. This is to prove that not only CAD software can be used in design but it can also be used in the medical field. The conversion of medical images to CAD form can simplify the images manipulation process for the purpose of learning or research [5].

Overall, this study will produce DICOM images that have been converted to a Data Transfer Drawing (DXF) format. This study may be extended to produce software that can convert DICOM data directly to CAD for the purpose of medical images manipulation to further prove that the $\mathrm{CAD}$ application is not only useful in product designing but also in medical imaging.

\section{RESEARCH BACKGROUND}

DICOM is a standard format used in the medical field for sharing and viewing of medical images such as Computed Tomography Scan (CT SCANS), MRI and ultrasound. DICOM standard was developed by NEMA with the collaboration of ACR. A DICOM file has a header which store patient patient's information such as name, type of scan and the image data itself. DICOM format is able to avoid the possibility of errors if patient's scanned image information and data are kept separately such as the medical image and analyze format. The Analyze format stores image data format (*. img) and header (*. hdr) in separate files. Another difference between DICOM file and the Analyze file is that the DICOM image file can be compressed to shrink the size, for example, converted into JPEG and TIFF format [6].

The purpose of compression being done is to reduce the size of files so that the memory space usage can be reduced and also to speed up file transfers. Besides these advantages, there are also disadvantages as it affects the quality of the image [1]. There are two types of basic compression algorithm and they are lossy algorithm and lossless algorithm. Example of lossless algorithm compression is the GIF format (CompuServe Graphics Interchange Format). This format is often used but it is limited to only 256 colors. For lossy algorithm, the most commonly used format is JPEG (Joint Photographic Expert Group). It supports 24-bit images that contain millions of colors. The compressed image does not lose the information quality if only the compression process is kept to a minimum.

CAD is used to design and develop products that will be used by consumers or products used to develop other products [7]. It is an extensive use of computer-based tools and systems to model two or three dimensional physical object. CAD was introduced to assist designers in making designing work much easier and faster than sketching on paper. CAD has become more sophisticated and engineers or designers can see the real picture of the product to be developed in three-dimensional model [2]. CAD development has also led to the development of Computer Aided Manufacturing (CAM) which was introduced into the production environment. It allows data input in the CAM system to create programmed machines that can automate software processes that were previously done manually.

CAD was initially created for the engineering designers, but because of its potential use in other areas, particularly in mapping area, has resulted in many changes and now there are a lot of file format and extension such as STL, DXF, DWG, IGES, SAT, STEP, ISFF, XGL and many more. It is normal for CAD packages to support 3D solid modelling, and some packages also offer the rendering and animation function. CAD packages enable object in the drawing to be linked to the internal database. The ability to import raster images as backgrounds or as rendering textures is common for any packages.

With the advantages provided by CAD packages, it is very important that medical images (DICOM) can be converted to $\mathrm{CAD}$ format. This allows medical images to be manipulated in changing the image to a $3 \mathrm{D}$ format, making the image rotation, etc.

\section{METHOD}

In general, this study involves three main process, DICOM images, DICOM image conversion to JPEG and JPEG conversion to CAD format. CAD format will enable better manipulation of the images. Figure 3 shows the conversion method of DICOM images to CAD. 


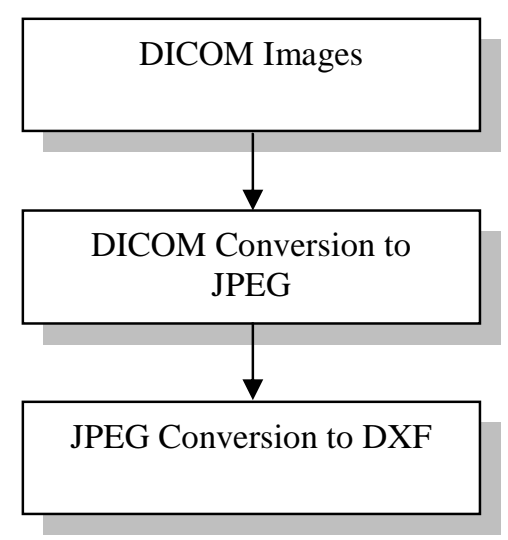

Fig. 3 DICOM image conversion process to CAD

\section{A. DICOM Image Conversion to Jpeg Format}

In this study, a DICOM image with the file extension *.dcm with a patient's hip bone is used. DICOM data display can be viewed by using Medweb Software (PPUKM, 2009) as in figure 4.The image is then converted into JPEG using Medweb software as in figure 5. This conversion is also referred to as compression for file size is smaller and the header or part of the store information is lost. Size of the image compression can be selected depending on user requirements. The less resolution is used, the less the quality of compressed images [9]. An image of patients with hip joint has been converted to JPEG format can be viewed as in figure 6 .

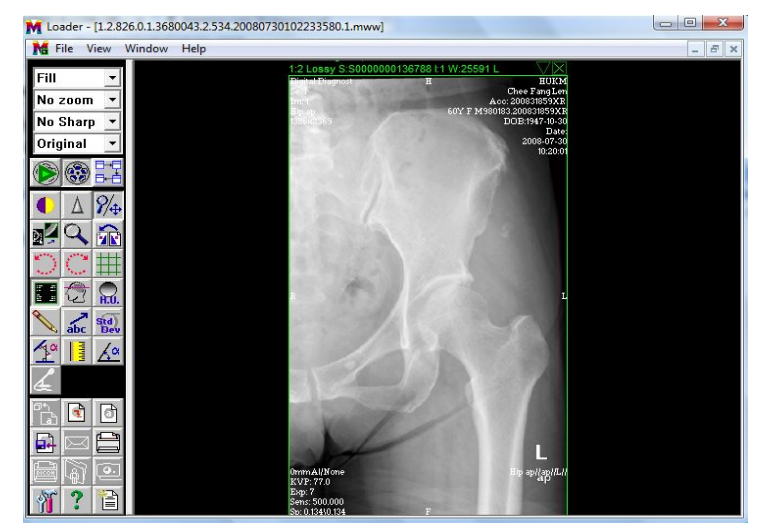

Fig. 4 Medweb software

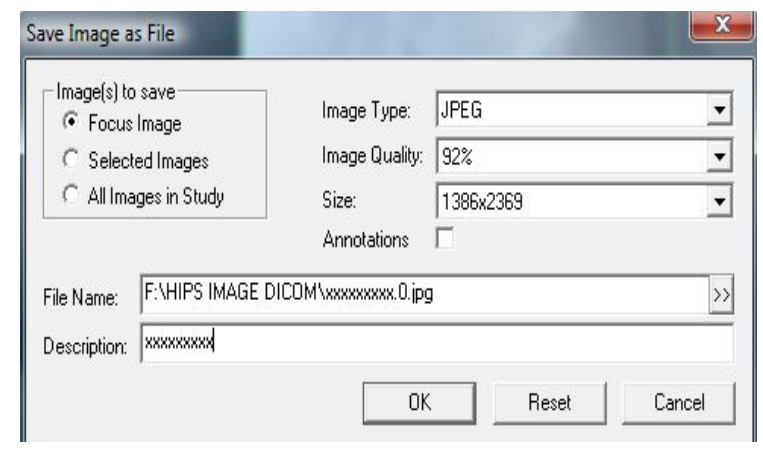

Fig. 5 Convert DICOM to JPEG

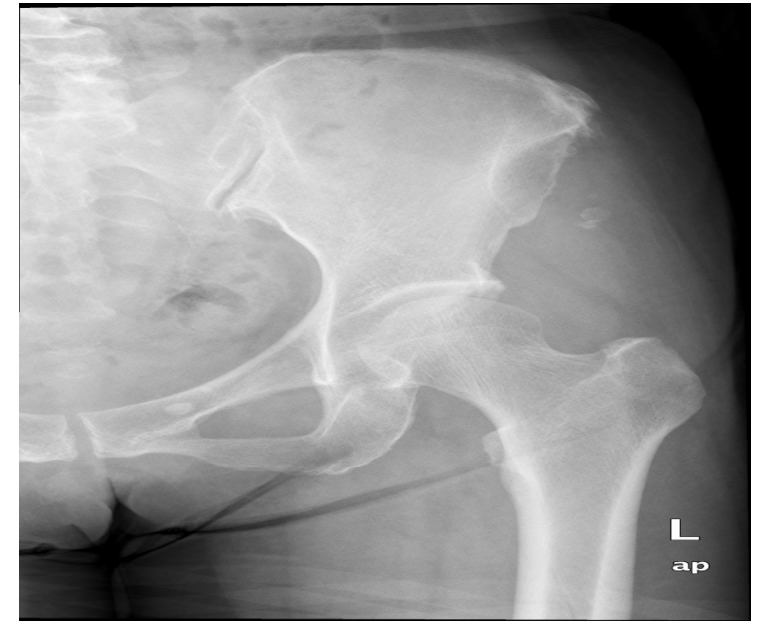

Fig. 6 DICOM image in JPEG format

\section{B. JPEG Image Conversion to CAD Format}

JPEG format that have been converted from DICOM images will then be converted into DXF format using Img2CAD software as in figure 7 below. Image2CAD is a handy tool for converting raster image to DXF file, especially useful for title block's logo importing. This software is often used by designers to convert hand drawings to CAD format.

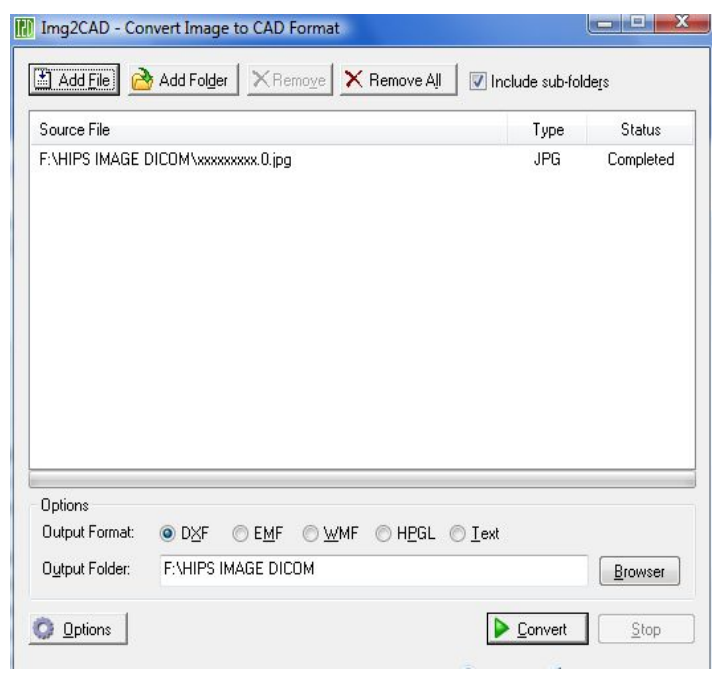

Fig. 7 Convert JPEG to CAD format

\section{RESULTS \& DISCUSSION}

The display result on AutoCAD 2009 is shown in Figure 8. This proves that it is possible for CAD software to be used in medical imaging technology. This is particularly useful for as medical images can be easily changed and animated for learning and research purposes. Image manipulation can be done with a geometric transformation (rotation, translations etc.) and give out colours to produce a display that can provide more information for research purposes [7]. With the rapid development on $\mathrm{CAD}$, medical images can be transformed into a three dimensional (3D) format. Images in 
the form of 3D helps the medical experts in conducting study on the patient more accurately.

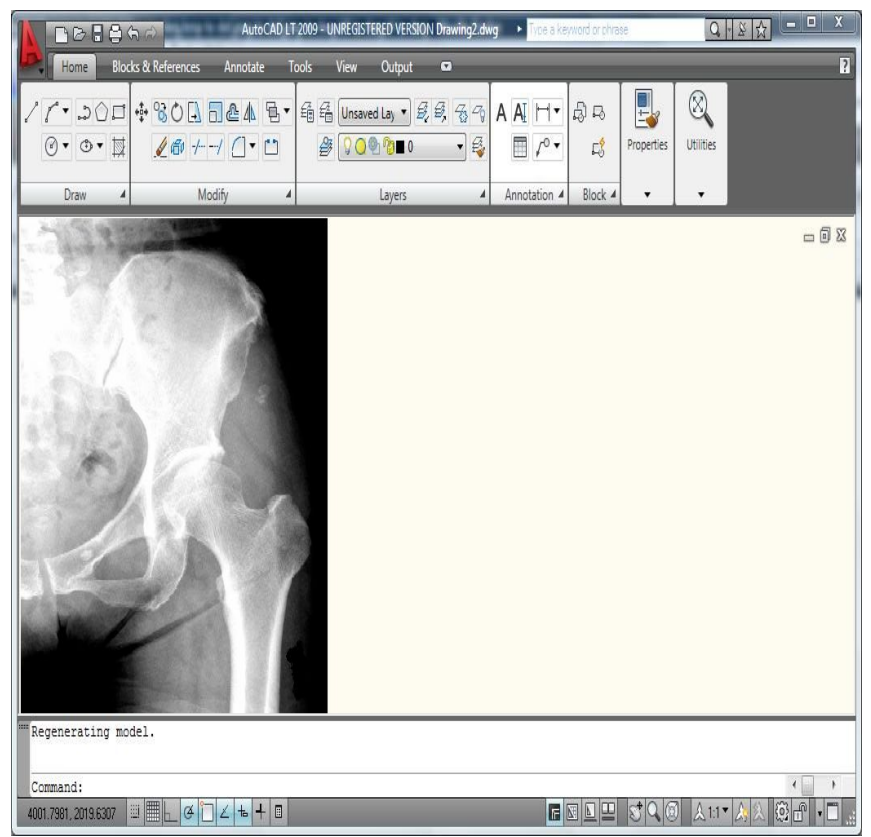

Fig. 8 DICOM image display by AutoCAD

\section{CONCLUSION}

This study may be something new in which it is an idea to apply the CAD software in the presentation of medical images (DICOM). Until now, CAD is widely used only in the fields that involve with designing of the manufacturing industry products. This study involves the conversion of the images in the DICOM files, but does not include the image information itself on the header. This means that only images and not the images information (e.g. name of patient) that can be transferred. In general, this is particularly a very interesting study because it integrates ideas from two very different fields of medicine and design. Medical images in CAD format can be converted into three-dimensional (3D) form. The use of 3D images will help medical experts to diagnose the patient's condition more easily because the 3D images can be viewed in better and clearly than the twodimensional images (2D) [10].

\section{ACKNOWLEDGMENT}

This research project conducted in collaboration with Dr. Abdul Yazid Mohd Kassim and Dr. Hamzaini Abd Hamid from the Department of Orthopedics and Traumalogy, Medical Centre of Universiti Kebangsaan Malaysia. This department has provided medical image data (DICOM) to be used in this research. This research also funded by University Grant UKM-OUP-ICT-34-171/2009 and UKMGUP-TMK-07-01-035.

\section{REFERENCES}

[1] Digital Imaging and Communication on Medicine. National Electrical Manufacturers Association (NEMA) std, 2006.

[2] S. Riza, Mengenali Rekabentuk Berbantukan Komputer (RBK). Edisi Kedua. Universiti Kebangsaan Malaysia, Bangi, Malaysia, 2002.

[3] C. Russ, The Image Processing Handbook. Boca Raton, Fla.: CRC Press, 2002.

[4] J. Fauquex, "Color Management for DICOM Images Considered as TIFF 16," Display Technology, vol. 4, pp. 410-414, 2008.

[5] L.W. Kit, "Permodelan Anatomi Manusia Secara Tiga Dimensi Berdasarkan Imej Perubatan DICOM Dalam Persekitaran CAD," Msc thesis, Universiti Kebangsaan Malaysia, Bangi, Malaysia, 2007.

[6] R.N.J. Graham, R.W. Perriss, A.F. Scarsbrook, "DICOM Demystified: A Review of Digital file formats and their use in radiological practice", Clinical Radiology, vol. 60, pp. 1133-1140, 2005.

[7] S. Riza, A. Yuwaldi, Rekabentuk Berbantukan Komputer. Universiti Kebangsaan Malaysia, Bangi, Malaysia. 2002.

[8] M. Howison, C.H. Sequin, "CAD Tools for Creating Space-filling 3D Escher Tiles," Computer Aided Design and Applications, vol. 6 (6), pp. 737-748, 2009.

[9] Y. T. Wu, F. Shih, J. Shi, “Atop-down region dividing approach for image segmentation", Pattern Recognition, vol. 41 , pp. 1948 - 1960, 2008.

[10] D. Gur, J.H. Sumkin. "CAD in Screening Mammography," American Journal of Roentgenology, vol.187, pp.1474, 2006. 